\title{
Puerperal brain cryptococcoma in an HIV-negative woman successfully treated with fluconazole: a case report
}

\author{
José Edward Hagan ${ }^{[1],[2], ~ J e s a ̂ n g e l i ~ S o u s a ~ D i a s ~}{ }^{[3],[4], ~ J o s e ́ ~ C a e t a n o ~ V i l l a s b o a s-B i s n e t o ~}{ }^{[2],}$ \\ Melissa Barreto Falcão ${ }^{[3]}$, Albert Icksang Ko ${ }^{[1],[2]}$ and Guilherme Sousa Ribeiro ${ }^{[2],[5]}$
}

[1]. Department of Epidemiology of Microbial Diseases, Yale School of Public Health, New Haven, CT, USA. [2]. Centro de Pesquisas Gonçalo Moniz, Fundação Oswaldo Cruz, Ministério da Saúde, Salvador, BA. [3]. Hospital Couto Maia, Secretaria de Saúde do Estado da Bahia, Salvador, BA. [4]. Faculdade de Medicina da Bahia, Universidade Federal da Bahia, Salvador, BA. [5]. Instituto de Saúde Coletiva, Universidade Federal da Bahia, Salvador, BA.

\begin{abstract}
Cryptococcus spp. cerebral abscesses are uncommon in immunocompetent subjects. The recommended induction treatment is the administration of amphotericin B plus flucytosine combined with resection for lesions $\geq 3 \mathrm{~cm}$. In this paper, we describe an HIV-negative woman diagnosed with a large cryptococcoma in the immediate postpartum period. The lesion was not resected, and due to amphotericin B intolerance, she received an extended course of fluconazole monotherapy. There was no disease recurrence during the 4 years of follow-up. The abrupt onset of her symptoms following delivery suggests that she developed a postpartum immune reconstitution syndrome. This case also demonstrates that in specific situations fluconazole monotherapy can be attempted in immunocompetent patients with cryptococcoma.
\end{abstract}

Keywords: Cryptococcus. Immune reconstitution inflammatory syndrome. Treatment.

\section{INTRODUCTION}

Most cases of central nervous system (CNS) infection caused by Cryptococcus spp. manifest as meningitis. In sporadic cases, Cryptococcus spp. CNS infection can result in the formation of a cryptococcoma, which can resemble a neoplastic mass. Cryptococcal disease typically occurs in immunocompromised individuals. Although Cryptococcus gattii infections have been increasingly recognized in immunocompetent hosts ${ }^{1-3}$, the incidence of brain cryptococcoma remains very low in healthy individuals ${ }^{3}$. We report a novel case of a large CNS cryptococcoma in an human immunodeficiency virus (HIV)-negative woman that was successfully treated with fluconazole. Her symptoms developed during the immediate postpartum period, suggesting a presentation exacerbated by postpartum immune reconstitution.

\section{CASE REPORT}

In December 2006, a 25-year-old female, who worked as a primary school teacher in a small city in northeast Brazil, was referred to a neurologist with complaints of progressively worsening right-sided paresthesias and muscle weakness. She denied having

Address to: Dr. Guilherme Sousa Ribeiro. Instituto de Saúde Coletiva/UFBA. Rua Basílio da Gama s/n, Campus Universitário Canela, 40110-040 Salvador, BA, Brasil.

Phone: 5571 3283-4707

e-mail: gsribeiro@ufba.br

Received 15 October 2013

Accepted 22 January 2014 any other symptoms, including pulmonary complaints. Her current symptoms began abruptly in the week following an otherwise uneventful pregnancy and spontaneous vaginal delivery of a healthy baby. She was previously healthy, but recalled that six years prior she had experienced a two-week episode of right-sided paresthesias and headache that resolved spontaneously.

The initial physical exam was unremarkable except for wordfinding difficulties and weakness and numbness of the rightsided extremities. There was no papilledema on fundoscopic examination, and routine laboratory studies were normal. A computed tomography scan of the brain without contrast revealed a heterogeneous mass that was further evaluated by magnetic resonance imaging (MRI). Post-gadolinium infusion images revealed a $3 \mathrm{~cm} \times 2 \mathrm{~cm}$ heterogeneously enhancing lesion arising in her left thalamus with a well-defined rim of enhancement and central hypointense areas suggestive of necrosis (Figure 1A and 1B). Based on the radiographic appearance, the patient was presumptively diagnosed with glioblastoma multiforme. While awaiting surgical evaluation, prophylaxis with dexamethasone and phenytoin was initiated, resulting in some initial improvement in her symptoms. A non-stereotactic biopsy of the mass was performed without resection. The pathological examination revealed numerous budding encapsulated organisms consistent with Cryptococcus sp. without granulomata or multinucleated giant cells (Figure 1C). The tissue was not sent for microbiologic studies due to a primary suspicion of CNS malignancy; therefore, the species and serotype could not be determined.

A chest X-ray revealed no evidence of active lung disease, scarring, or calcified granulomata to suggest prior pulmonary cryptococcosis. An enzyme-linked immunosorbent assay (ELISA) test for HIV I/II was negative. Additional testing for inherited immunosuppression was not performed. A routine 


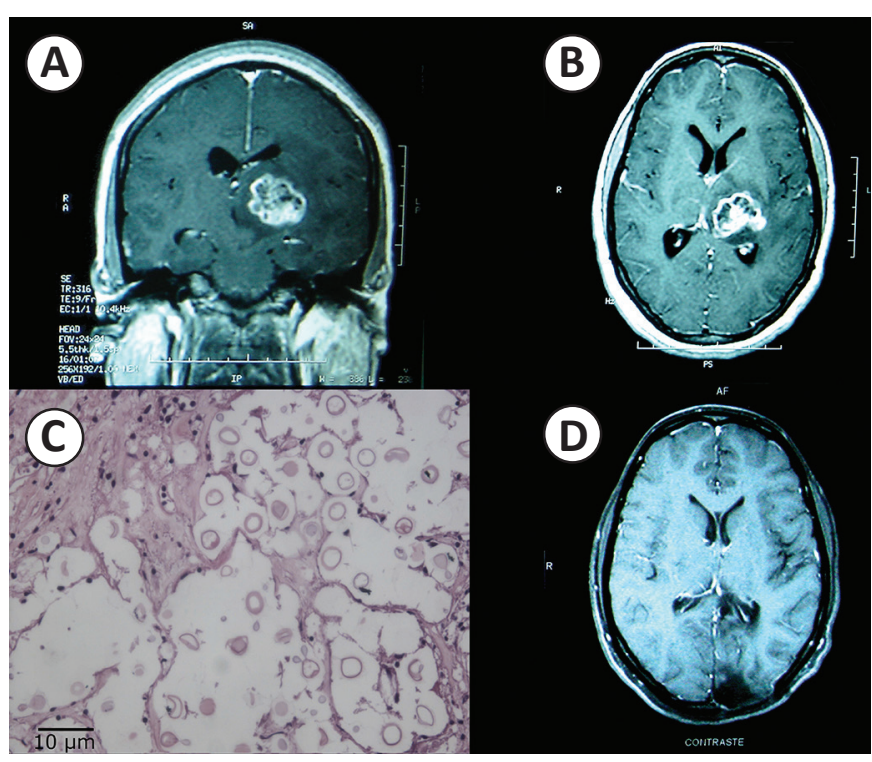

FIGURE 1 - Central nervous system cryptococcoma. T1-weighted postgadolinium coronal (A) and transverse (B) MRI images revealing a $3 \mathrm{~cm} \times 2 \mathrm{~cm}$ enhancing mass in the left thalamus with central hypointensity suggestive of necrosis. C: Hematoxylin and Eosin stain of brain biopsy tissue at 40x revealing numerous budding encapsulated organisms consistent with Cryptococcus species. D: T1-weighted post-gadolinium transverse magnetic resonance imaging (MRI) image revealing post-craniotomy changes but no evidence of an intra-parenchymal mass

peripheral blood culture was negative for bacteria and fungus. A cerebrospinal fluid (CSF) examination revealed no cells, glucose level of $58 \mathrm{mg} / \mathrm{dL}$ (normal $=50-80$ ), and protein level of $33 \mathrm{mg} / \mathrm{dL}$ (normal=15-45). An India ink stain was negative, and a CSF cryptococcal antigen latex agglutination assay (IMMY, Norman, Oklahoma, USA) was positive.

Dexamethasone therapy was discontinued, and treatment with amphotericin B was initiated due to a diagnosis of CNS cryptococcoma. Flucytosine was not commercially available in Brazil at the time. Her hospital course was immediately complicated by a pruritic body rash, severe hypokalemia and hypomagnesemia, and ventricular arrhythmia; all of these symptoms resolved after the withdrawal of amphotericin B. She was, therefore, treated with fluconazole, $800 \mathrm{mg} /$ day i.v. for 2 months, which was well tolerated until her discharge from the hospital. The patient's symptoms resolved over 17 months of continuous oral fluconazole therapy ( 8 months of $600 \mathrm{mg} /$ day and 9 months of $300 \mathrm{mg} /$ day), and a follow-up brain MRI showed complete resolution of the lesion (Figure 1D). The patient was followed for four years after her treatment, during which time she experienced mild residual neuromotor symptoms but displayed no evidence of disease recurrence.

\section{DISCUSSION}

Our patient was negative for HIV and did not have any prior or subsequent history of recurrent infection, suggesting that she did not have a significant undiagnosed humoral or cellular immunodeficiency. Although we could not determine the Cryptococcus species that caused her disease, our patient lived in the northeast region of Brazil, where Cryptococcus gattii accounts for $66 \%$ of the cryptococcal isolates ${ }^{4}$. In Brazil, $75 \%$ of the cryptococcal infections in HIV-negative patients are due to Cryptococcus gattii ${ }^{4}$, which is increasingly recognized worldwide as a cause of infection in immunocompetent hosts ${ }^{2,3}$. In these patients, cryptococcal disease is more likely to manifest as a cryptococcoma, as compared to immunosuppressed patients ${ }^{3}$. Nevertheless, the likelihood of a brain mass caused by Cryptococcus in immunocompetent individuals is extremely low compared with immunocompromised patients ${ }^{1,3}$.

Our patient's symptoms began within the first week after delivering a healthy baby. Although several cases of cryptococcal meningitis in immunocompetent pregnant women have been described ${ }^{5,6}$, to our knowledge, only two cases of cryptococcoma during pregnancy have been previously reported ${ }^{7,8}$. The presence of antecedent symptoms six years before this illness suggests the possibility of a prior infection that was spontaneously controlled until recrudescence and growth of the cryptococcoma during the relative immunosuppression of pregnancy, in which cellmediated immunity is particularly affected. Additionally, the abrupt onset of her symptoms following delivery may suggest an inflammatory response related to postpartum immune reconstitution. This inflammatory response occurs rapidly after delivery and may result in the unmasking of previously occult infectious diseases, such as mycobacterial infections, including tuberculosis; viral infections; and fungal infections, such as Cryptococcus. This phenomenon has been described in several case reports, in which symptoms of previously quiescent cryptococcal disease presented or significantly worsened during the late third trimester or immediate postpartum period ${ }^{9}$.

The recommended therapy for CNS cryptococcoma includes induction therapy with amphotericin B and flucytosine for at least 6 weeks, followed by 6-18 months of consolidation therapy with fluconazole. Surgical excision and debulking are indicated as adjunct therapies for lesions that are greater than or equal to $3 \mathrm{~cm}^{10}$. Our patient was intolerant of amphotericin $B$ and did not undergo surgical resection but was successfully treated with 2 months of i.v. and 17 months of oral fluconazole. Fluconazole monotherapy is effective for cryptococcal meningitis in immunocompetent patients ${ }^{11}$ and has been successfully used without surgical resection in both invasive pulmonary and thyroid cryptococcoma ${ }^{12,13}$. However, fluconazole monotherapy without surgical resection has not been previously described in the successful treatment of CNS cryptococcoma. Our patient had complete resolution of the brain lesion in a follow-up MRI and remained free of disease recurrence for 4 years of follow-up. This finding suggests that extended fluconazole monotherapy can be attempted in immunocompetent patients with cryptococcoma caused by a susceptible organism who are intolerant of amphotericin B and who are not good candidates for surgical resection.

Finally, this case illustrates the potential of CNS cryptococcoma to be mistaken for glioblastoma multiforme and other intracranial neoplasms and also reveals the importance of considering fungal infection, including cryptococcoma, in the differential diagnosis of a CNS mass in immunocompetent pregnant women. 
Ethics approval to publish this case report was granted by the Hospital Couto Maia Ethics Committee. The patient provided written informed consent to have her case details published.

\section{ACKNOWLEDGMENTS}

The authors thank the Hospital Couto Maia, Bahia Secretary of Health for providing the infrastructure for the care of the patient.

\section{FINANCIAL SUPPORT}

This work was supported by the National Institutes of Health through the Fogarty International Clinical Research Scholars and Fellows Program at Vanderbilt University (R24 TW007988) and the American Relief and Recovery Act, and through grants, NIAID R01 AI052473 and FIC D43 TW00919.

\section{REFERENCES}

1. Li Q, You C, Liu Q, Liu Y. Central nervous system cryptococcoma in immunocompetent patients: A short review illustrated by a new case. Acta Neurochir 2010; 152:129-136.

2. Centers for Disease Control and Prevention (CDC). Emergence of Cryptococcus gattii- Pacific Northwest, 2004-2010. MMWR Morb Mortal Wkly Rep 2010; 59:865-868.

3. Chen S, Sorrell T, Nimmo G, Speed B, Currie B, Ellis D, et al. Epidemiology and host- and variety-dependent characteristics of infection due to Cryptococcus neoformans in Australia and New Zealand. Clin Infect Dis 2000; 31:499-508.
4. Nishikawa MM, Lazera MS, Barbosa GG, Trilles L, Balassiano BR, Macedo RCL, at al. Serotyping of 467 Cryptococcus neoformans isolates from clinical and environmental sources in Brazil: Analysis of host and regional patterns. J Clin Microbiol 2003; 41:73-77.

5. Costa ML, Souza JP, Oliveira Neto AF, Pinto e Silva JL. Cryptococcal meningitis in HIV negative pregnant women: Case report and review of literature. Rev Inst Med Trop Sao Paulo 2009; 51:289-294.

6. Pereira CAP, Fischman O, Colombo AL, Moron AF, Pignatari ACC. Neurocriptococcose durante a gravidez. Revisão da literatura. Relato de dois casos. Rev Inst Med Trop Sao Paulo 1993; 35:367-371.

7. Nucci A, Maciel Jr JA, Queiroz LS, Montenegro MA, Carvalho RB. Pseudocystic form of neurocryptococcosis in pregnancy: case report. Arq Neuropsiquiatr 1999; 57:678-682.

8. Mitchell DH, Sorrel TC, Allworth AM, Heath CH, McGregor AR, Papanaoum K, et al. Cryptococcal disease of the CNS in immunocompetent hosts: Influence of cryptococcal variety on clinical manifestations and outcome. Clin Infect Dis 1995; 20:611-616.

9. Singh N, Perfect JR. Immune reconstitution syndrome and exacerbation of infections after pregnancy. Clin Infect Dis 2007; 45:1192-1199.

10. Perfect JR, Dismukes WE, Dromer F, Goldman DL, Graybill JR, Hamill RJ, et al. Clinical practice guidelines for the management of cryptococcal disease: 2010 update by the Infectious Diseases Society of America. Clin Infect Dis 2010; 50:291-322.

11. Yamaguchi $\mathrm{H}$, Ikemoto $\mathrm{H}$, Watanabe $\mathrm{K}$, Ito A, Hara $\mathrm{K}$, Kohno S. Fluconazole monotherapy for cryptococcosis in non-AIDS patients. Eur J Clin Microbiol Infect Dis 1996; 15:787-792.

12. Huang CJ, Yang MC, Ueng SH. Large cryptococcoma mimicking lung cancer in an HIV-negative, type 2 diabetic patient. J Thorac Imaging 2005; 20:115-117.

13. Kagan E, Madden MB, Parsons CH. Successful treatment of a locally invasive cryptococcoma mimicking primary thyroid cancer with fluconazole. Am J Med Sci 2010; 340:173-175. 